\title{
Actualisation des données tardiglaciaires du Jura méridional (Ain) inventaire matériel et bilan sanitaire des gisements
}

\section{Gérald Bereiziat}

\section{(2) OpenEdition}

\section{Journals}

Édition électronique

URL : http://journals.openedition.org/adlfi/1578

ISSN : 2114-0502

Éditeur

Ministère de la culture

Référence électronique

Gérald Bereiziat, « Actualisation des données tardiglaciaires du Jura méridional (Ain) inventaire matériel et bilan sanitaire des gisements », ADLFI. Archéologie de la France - Informations [En ligne], Rhône-Alpes, mis en ligne le 01 mars 2008, consulté le 19 avril 2019. URL : http:// journals.openedition.org/adlfi/1578

Ce document a été généré automatiquement le 19 avril 2019

(c) Ministère de la Culture et de la Communication, CNRS 


\title{
Actualisation des données tardiglaciaires du Jura méridional (Ain) inventaire matériel et bilan sanitaire des gisements
}

\author{
Gérald Bereiziat
}

Date de l'opération : 2008 (PT)

1 Nos connaissances des dernières populations de chasseurs-cueilleurs venus occuper le versant méridional du Jura au sortir de la dernière grande glaciation se sont construites autour de diverses investigations menées depuis plus d'un siècle. Aux premiers travaux empiriques réalisés entre 1880 et 1920 ont succédé des interventions davantage minutieuses et méthodiques de 1950 à nos jours. Cette longue histoire des recherches a permis de récolter une importante documentation mais de valeur souvent inégale en raison des limites imposées par les méthodes d'excavation employées et des dispersions successives des collections. Cette situation, qui a d'ailleurs longtemps ralenti la mise en place d'un cadre chrono-culturel régional, liée à un essoufflement des activités sur le terrain durant les deux dernières décennies, provoquent aujourd'hui une certaine paralysie de nos acquis et de la considération même du statut de certains sites qui restent encore trop tributaires de l'ancienneté de leur trouvaille.

2 Face à ce constat, ce projet avait pour ambition de proposer, par un travail de sériation et d'état des lieux des gisements, un regard actuel sur la version quantitative et qualitative des témoignages de la présence des hommes tardiglaciaires dans cette zone géographique.

3 Cette "prospection " permet dès à présent d'avoir une réflexion commune sur la place des collections au sein de notre système d'exploitation, et d'aborder la question de la sauvegarde et de la gestion des gisements désertés depuis les dernières investigations. 


\section{Cadre chrono-culturel du projet}

4 Plaque tournante entre le bassin rhodanien au sud et les plateaux suisses et jurassiens au nord, le Jura méridional a connu une occupation relativement dense et continue au sortir de la dernière période glaciaire. Tous situés en grotte ou abris sous-roche, les treize gisements répertoriés se répartissent entre le nord et le sud de la région, pour la plupart dans des zones de passages naturels, au contact des cours d'eau. (Fig. $n^{\circ} 1$ : Localisation des sites retenus pour le projet)

5 Le Magdalénien moyen (Dryas ancien inférieur, vers 14000 BP) est identifié, dans sa phase terminale, pour les niveaux d'occupations de La Colombière et de La Croze.

6 Les autres sites se calent tous dans la biozone du Bölling, que l'on peut sérier en plusieurs phases:

7 - en limite de la fin du Dryas ancien et début du Bölling, entre 13000 BP et 12700 BP pour les Romains, l'abri Gay (foyer fd2), les Hoteaux (couche g), Thuys II et Thuys I.

8 - la première moitié du Bölling, entre $12600 \mathrm{BP}$ et $12000 \mathrm{BP}$, pour la couche 2 de La Chênelaz et, de par un diagnostic de l'industrie lithique pour les sites de La Bonne Femme, Pugieu et La Grand'Baille.

9 - la fin du Bölling (voire début du Dryas moyen), entre 12400 BP et 12000 BP pour La Raillarde, l'abri Gay (f2a/b), l'Abbaye I.

\section{Inventaire du matériel : entre richesse et dispersion...}

10 Cette documentation se caractérise tant par sa richesse que par sa diversité. Les manifestations artistiques tels que les galets gravés de La Colombière et de La Croze, la sépulture des Hoteaux, ou la finesse des éléments de parure de la grotte des Romains témoignent en effet d'une période exceptionnellement fertile, et nul doute qu'un regroupement de l'ensemble des séries préhistoriques de l'Ain dispersées dans divers organismes publics ou privés ferait de cette région un des pôles les plus importants du Tardiglaciaire pour l'est de la France.

11 Malheureusement, cette richesse est trop souvent masquée par le morcellement des collections. Plusieurs sites soulignent des lacunes importantes qui influent sur nos connaissances et ne permettent pas d'aborder avec précision le cadre chrono-culturel de leurs implantations. Cette situation peut se comprendre pour les produits des fouilles anciennes récoltés très souvent par des amateurs ou des membres de sociétés savantes régionales entre la fin du XIX ${ }^{\mathrm{e}}$ s.et le début du XXe $\mathrm{x}$. mais, aujourd'hui encore, la gestion des collections actuelles reste un problème. Elles se trouvent en effet confrontées à l'attente des études de fonds et à l'acceptation d'une destination précise.

12 Le vœu le plus cher serait de pouvoir un jour centraliser tout ce matériel et d'avoir un équilibre entre sa représentativité et se mise en valeur, et assister enfin à une vraie politique commune. 


\section{Etat des lieux des gisements}

Le deuxième objectif de ce travail concernait l'intégrité actuelle des sites. Nos observations ont intéressé autant l'aspect général (accessibilité au site, importance du couvert végétal, dangerosité, etc.) que la surface excavée (intégrité de la coupe stratigraphique, fonctionnalité du carroyage encore en place, intégrité de la structure protectrice, etc.) et l'avenir du site (potentiel archéologique, place dans le patrimoine régional ...).

Les conclusions de notre passage sur 9 des 13 sites répertoriés dans le Jura méridional sont partagées entre un sentiment de fatalité face à la désertification des lieux et l'espoir de pouvoir exploiter les informations encore présentes dans le sol.

Devant le constat offert par chaque gisement, nous avons émis plusieurs propositions consistant à valoriser leur position actuelle, tant d'un point de vue de la conservation et de la protection, que de la capacité à exploiter toutes les possibilités de nouvelles recherches. Nous sommes conscients que toutes nos requêtes seront difficiles à mettre en place, mais un programme pluriannuel clairement établi permettrait de contrôler leur réalisation.

Enfin, en guise d'épilogue, l'insertion de ces gisements dans un parcours culturel serait le point d'orgue de ce programme de réhabilitation et promouvrait ainsi la diffusion d'un patrimoine local encore peu connu du grand public.

\section{ANNEXES}


Fig. $\mathrm{n}^{\circ} 1$ : Localisation des sites retenus pour le projet

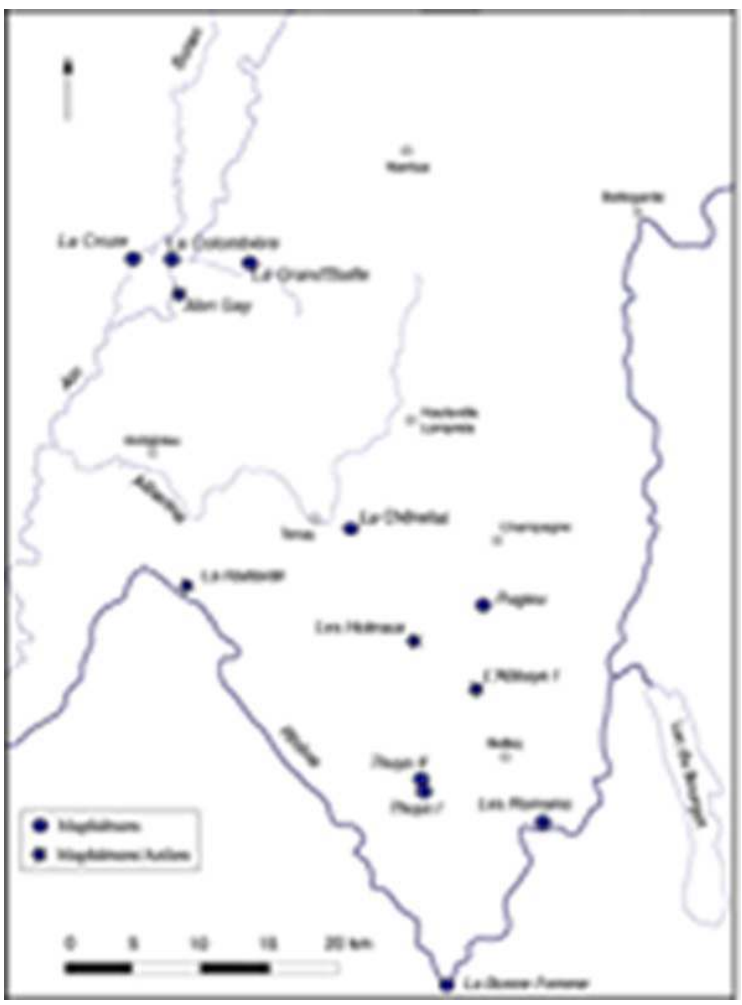

Auteur(s) : Bereiziat, Gérald (chercheur bénévole). Crédits : Bereiziat (2008)

INDEX

operation Prospection thématique (PRT)

Thèmes : conservation de site, conservation-restauration, galet gravé, grotte, inventaire, parure, patrimoine, sépulture, stratigraphie

Index chronologique : Tardiglaciaire

Index géographique : Rhône-Alpes, Ain (01) 